\title{
Pueblos Indígenas, Estados y Educa- ción Superior en América Latina. Modalidades de colaboración, logros, problemas, desafíos y conflictos ${ }^{1}$ Povos Indígenas, Estados e Ensino Superior na América Latina. Modalidades de colaboração, conquistas, problemas, desafios e conflitos
}

\author{
Daniel Mato*
}

Resumen: Este texto ofrece un panorama general de las diversas modalidades de colaboración entre pueblos indígenas y universidades y otros tipos de instituciones de Educación Superior actualmente en curso en América Latina, así como de las iniciativas propias y autónomas de algunas organizaciones indígenas en este campo. Adicionalmente, señala sus principales logros, problemas, desafíos, y conflictos, los cuales generalmente se plantean con los Estados, a través de sus organismos del área de educación.

Palabras clave: pueblos indígenas, estados, educación superior.

Resumo: Este texto fornece uma visão geral das várias formas de colaboração entre os povos indígenas, universidades e outras instituições de ensino superior em curso na América Latina, bem como iniciativas próprias e independentes de algumas organizações indígenas neste domínio. Além disso, assinala suas principais realizações, problemas, desafios e conflitos, que geralmente surgem com os Estados, por meio de suas agências na área de educação.

Palavras-chave: povos indígenas, estados, ensino superior.

\footnotetext{
${ }^{1}$ Este artículo es una versión ampliada de la conferencia inaugural ofrecida en el "V Seminário: Povos Indígenas e Sustentabilidade: do campo ao campus e do campus ao campo: trajetórias de saberes", organizado por el Núcleo de Estudos e Pesquisas das Populações Indígenas (NEPPI/UCDB), Projeto Rede de Saberes (UCDB, UFMS, UEMS e UFGD), y el Observatório da Educação Escolar Indígena, Universidade Católica Dom Bosco (UCDB), Campo Grande, Mato Grosso do Sul, Brasil, 23-25/09/2013.
}

* CONICET - UNTREF E-mail:dmato2007@gmail.com 
En las últimas dos décadas, la mayoría de las sociedades latinoamericanas han logrado mejorar la calidad de sus democracias respecto de algunas inequidades que afectan particularmente a pueblos indígenas y afrodescendientes. Aunque aún insuficientes, estas mejoras son importantes y su fortalecimiento y profundización debería interesar no solo a esos pueblos, sino a todos los sectores de las respectivas sociedades nacionales. Porque, incluso más allá de cualquier consideración ética y de vigencia efectiva de las respectivas Constituciones nacionales, estas sociedades se beneficiarían de la incorporación plena de la multiplicidad de conocimientos y otros recursos propios de la diversidad cultural característica de cada una de ellas.

La Educación Superior de la región ha venido jugando un tímido papel en estos procesos. No obstante, existe ya una cierta cantidad de experiencias valiosas en desarrollo. Algunas de ellas se han desarrollado a través de diversos tipos de modalidades de colaboración entre pueblos indígenas y universidades y otras Instituciones de Educación Superior (IES). Otras son iniciativas propias y autónomas de organizaciones indígenas. Finalmente, otras son iniciativas de los Estados, generalmente desarrolladas en respuesta a demandas del movimiento indígena. En las próximas páginas se presenta un panorama general de esa diversidad de modalidades. Adicionalmente, se señalas sus principales logros, problemas, desafíos, y conflictos, estos último generalmente se plantean entre organizaciones indígenas y organismos gubernamentales del área de educación (secretarías, ministerios, agencias de evaluación y acreditación de programas e instituciones, entre otros, según el caso).

Derivadas de la herencia colonial, las inequidades que afectan a pueblos indígenas y afrodescendientes aún están fuertemente enquistadas en las sociedades latinoamericanas contemporáneas. Más allá de transformaciones que no carecen de importancia, su continuidad puede observarse en algunas leyes y políticas públicas, así como en las prácticas de instituciones y actores sociales. Estas inequidades frecuentemente pasan inadvertidas para la mayor parte de la ciudadanía, son parte del "sentido común" establecido, de los imaginarios sociales prevalecientes. Por eso, las mencionadas mejoras recientes en la calidad de las democracias latinoamericanas han sido impulsadas principalmente por organizaciones, dirigentes, e intelectuales, de los pueblos indígenas y afrodescendientes de numerosos países de la región. En la mayoría de estos países, su accionar se ha visto complementado por el de algunos otros sectores sociales de esas mismas sociedades, incluyendo organizaciones civiles de diversos tipos, fundaciones privadas, universidades y otros tipos de IES, así como autoridades, estudiantes, docentes, investigadores y egresados de éstas. También han recibido significativo apoyo de algunas organizaciones no gubernamentales y fundaciones privadas de otros países, y también de algunos organismos intergubernamentales y agencias 
de cooperación internacional. En algunos casos, estas iniciativas han contado también con la receptividad y/o adhesión más o menos proactiva de algunos dirigentes políticos, parlamentarios y autoridades ejecutivas de los respectivos países. Estos cambios se han visto favorecidos también por un cierto "clima de época" y horizonte ético-político que encuentra expresión en un significativo conjunto de tratados y convenciones internacionales, a algunos de los cuales se hará referencia en este capítulo.

Las mejoras en cuestión se han expresado en importantes reformas constitucionales, leyes y políticas públicas, así como en algunos cambios favorables en las prácticas concretas de algunos agentes sociales. Aunque valiosos, los avances logrados en las constituciones nacionales, leyes y políticas resultan aún insuficientes para reparar los efectos de agresiones, siglos de racismo y desconocimiento de la propia historia. Pero, además, en la práctica, estos avances muchas veces se ven menoscabados debido a la persistencia de diversas formas de racismo (frecuentemente solapado, o inconsciente), la "naturalización" de situaciones inequitativas, o al menos la indiferencia hacia ellas, así como a intereses económicos domésticos e internacionales, y al eurocentrismo e ignorancia respecto de la propia historia de diversos sectores sociales de estos países. Estos y otros factores hacen que los avances logrados en las constituciones nacionales de varios países se expresen insuficientemente en las leyes vigentes, y/o en sus reglamentaciones y formas concretas de aplicación, y/o en políticas públicas efectivas, su puesta en práctica y asignaciones presupuestarias para las mismas.

El educativo es uno de los campos en los cuales pueden observarse algunos avances significativos, incluso aunque aún sean insuficientes. Estos se registran especialmente respecto de acceso de dichos pueblos a educación básica y en ocasiones media y superior, aunque frecuentemente resultan más significativos en el nivel normativo que en el de las realizaciones prácticas. Las realizaciones son incluso menos significativas en materia de educación intercultural bilingüe, que constituye un factor crítico para asegurar cualquier avance que pueda lograrse en educación superior, pero que a la vez depende de que universidades y otros tipos de IES formen docentes especializados en EIB, que sean hablantes de las respectivas lenguas.

En el caso de la educación superior, si bien ha habido algunos avances respecto de acceso, permanencia y graduación de estudiantes de dichos pueblos, éstos aún resultan marcadamente insuficientes. Pero, los avances han sido incluso menos significativos si nos planteamos el tema en términos de la pertinencia del sistema de educación superior de estos países respecto de la diversidad cultural propia de cada uno de ellos; e incluso y de manera más general respecto de las necesidades, problemas y propuestas de amplios sectores de población, independientemente de su adscripción étnica. 
Es decir, si nos planteamos el asunto en términos de "interculturalización" de la educación superior, entendida como la inclusión de los conocimientos y modos de producción de conocimientos, modos de aprendizaje, lenguas, historias, visiones de mundo, y proyectos de futuro de los pueblos indígenas y afrodescendientes, en los planes de estudio de todas las universidades y otros tipos de IES, según sea pertinente para las diversas formaciones profesionales.

Antes de entrar en materia es necesario apuntar brevemente algunas referencias demográficas, históricas y normativas

\section{Aspectos demográficos, históricos y normativos}

Aunque la importancia y significación social, cultural y política de los pueblos indígenas no puede reducirse a aspectos cuantitativos, es necesario tener presentes algunos indicadores demográficos que permiten apreciar su importancia numérica, especialmente en algunos países de la región.

Con base en las cifras censales más recientes de los respectivos países, a grandes rasgos cabe señalar que en Bolivia la población indígena representa aproximadamente el 50\% del total nacional, en Guatemala el 39\%, en Chile, México, Panamá y Perú representa entre el 11\% y el 16\%; en Colombia, Ecuador, Honduras y Nicaragua representa entre el $4 \%$ y el $8 \%$; en Argentina, Costa Rica, Paraguay y Venezuela representa entre el 1,5\% y el 2,5\%; en Brasil el 0,4\% y en El Salvador menos del 0,2\%; en todos los casos los porcentajes indicados lo son respecto de los respectivos totales nacionales. En números absolutos, en el caso de México se trata de más de 10 millones de personas, en los de Bolivia, Perú y Guatemala más de 4 millones en cada uno de ellos, en el de Chile casi 2 millones de personas, en los de Colombia y Ecuador más de un millón de personas en cada caso, en Argentina es casi 1 millón de personas, en Brasil más de 800 mil personas, y en Honduras, Nicaragua, Panamá y Venezuela se trata de entre 400 mil y 600 mil personas en cada uno de ellos (MATO, 2012). Las cifras y porcentajes antes mencionados deben considerarse mínimos, pues se trata de datos oficiales, los cuales en muchos casos han sido cuestionadas por las organizaciones indígenas de los respectivos países.

Para comprender las relaciones actuales entre pueblos indígenas y universidades, otras instituciones de educación superior y Estados en América Latina, es indispensable recordar algunos factores históricos que resultan especialmente significativos para el tema que nos ocupa.

Como se sabe, la historia de América ha sido marcada por la conquista y colonización europea, con masacres, despojos de territorio, desplazamientos y reorganización social y territorial de los pobladores originales de esta parte del mundo. Como parte de esos procesos, los pueblos indígenas americanos 
vivieron varias circunstancias que atentaron especialmente contra importantes elementos constitutivos de sus visiones de mundo. Uno de ellos es que sus religiones fueron prohibidas y fueron forzados a adoptar el catolicismo. Otro es que también sus lenguas fueron prohibidas, cuánto menos su uso en espacios públicos y especialmente en la escuela, cuando accedieron a ella. Otro tanto ocurrió con sus conocimientos ancestrales, particularmente los relativos al campo de la salud, que los colonizadores asociaban a la idea europea de "brujería". La fundación de las nuevas repúblicas en el siglo XIX de ningún modo acabó con estas condiciones. Los nuevos Estados continuaron muchas de esas prácticas y a través de sus políticas educativas y culturales desarrollaron imaginarios nacionales homogeneizantes, negadores de las diferencias. Un aspecto importante de la problemática político-social, educativa y epistemológica, es que aún hoy, en pleno Siglo XXI, esta historia de prohibiciones y exclusiones no ha acabado, sino que continúa bajo diversas formas en la Escuela y en las Universidades y otras IES "convencionales" ${ }^{2}$ contemporáneas. Otro aspecto importante es que tampoco han culminado el despojo de territorios, ni el racismo, ni las prácticas genocidas, ni la imposición de un cierto modelo de desarrollo. Por eso estos asuntos también forman parte de la agenda de relaciones entre pueblos indígenas, Estados, y universidades y otros tipos de IES, e inciden en el tratamiento que los Estados dan a las demandas y propuestas de comunidades y organizaciones indígenas en materia educativa educativas (no solo de nivel superior, sino de todos los niveles).

Al hacer este breve repaso sobre aspectos de contexto, también es necesario señalar que en la actualidad las constituciones y leyes de la mayoría de los países latinoamericanos reconocen a los pueblos indígenas derechos de idioma, educación, identidad y otros relacionados. Hasta el presente este reconocimiento está consagrado en las constituciones de dieciséis países latinoamericanos, que resulta conveniente enumerar: Argentina, Bolivia, Brasil, Colombia, Costa Rica, Ecuador, El Salvador, Guatemala, Honduras, Guyana, México, Nicaragua, Panamá, Paraguay, Perú y Venezuela (MATO, 2012).

A esto se agrega que catorce países latinoamericanos han ratificado el Convenio 169 de la Organización Internacional del Trabajo (OIT), son: Argentina, Bolivia, Brasil, Chile, Colombia, Costa Rica, Ecuador, Guatemala, Honduras, México, Nicaragua, Paraguay, Perú y Venezuela. Es necesario subrayar que, tal como lo dispone este Convenio y las constituciones nacionales de los países antes mencionados, los Estados que han ratificado este instrumento internacional están obligados a acatar sus regulaciones (MATO, 2012). No disponemos de espacio para entrar en muchos detalles, pero como mínimo debe señalarse que los artículos 22, 26 y 27 del Convenio 169 consa-

\footnotetext{
${ }^{2}$ En este texto se llama "convencionales" a las IES que no han sido diseñadas para responder a necesidades, demandas o propuestas de pueblos indígenas y afrodescendientes.
} 
gran derechos específicos a los pueblos indígenas en materia de "educación a todos los niveles" (art. 26), es decir también de educación superior. Además, en particular, el artículo 27 establece que

[Los programas de educación destinados a estos pueblos deben] responder a sus necesidades particulares, y deberán abarcar su historia, sus conocimientos y técnicas, sus sistemas de valores y todas sus demás aspiraciones sociales, económicas y culturales, así como que la autoridad competente deberá asegurar la formación de miembros de estos pueblos y su participación en la formulación y ejecución de programas de educación, con miras a transferir progresivamente a dichos pueblos la responsabilidad de la realización de esos programas, cuando haya lugar; [...]. Y finalmente estipula que] los gobiernos deberán reconocer el derecho de esos pueblos a crear sus propias instituciones y medios de educación, siempre que tales instituciones satisfagan las normas mínimas establecidas por la autoridad competente en consulta con esos pueblos. Deberán facilitárseles recursos apropiados con tal fin. (http:/ / www.ilo. org/public/spanish/region/ampro/lima/publ/conv-169/convenio. shtml; consultada: 12/06/2014).

No obstante, el Convenio 169 de la OIT no es el único instrumento internacional que establece regulaciones en la materia que nos ocupa. También lo hacen la Convención Internacional para la Eliminación de todas las Formas de Discriminación Racial (1965), la Convención Internacional sobre Derechos Económicos, Sociales y Culturales (1966), la Declaración de las Naciones Unidas sobre los Derechos de las Personas Pertenecientes a Minorías Étnicas, Religiosas y Lingüísticas (1992), la Declaración Universal de la UNESCO sobre Diversidad Cultural (2001), la Convención de la UNESCO sobre la Protección y Promoción de la Diversidad de las Expresiones Culturales (2005), la Declaratoria de Naciones Unidas de la Segunda Década de los Pueblos Indígenas 2005-2015, y la Declaración Universal sobre los Derechos de los Pueblos Indígenas (2007).

Sin embargo, un estudio realizado por el Instituto Internacional de la Unesco para la Educación Superior en América Latina y el Caribe (UNESCOIESALC), permitió constatar que en la mayoría de los casos, las mencionadas regulaciones del Convenio Nro. 169 de la OIT, de cumplimiento obligatorio para los Estados que lo han ratificado, así como las normas constitucionales y legales establecidas en la materia, aún tienen escasos efectos prácticos en los países en cuestión. Lo establecido en estas normas jurídicas se expresa muy parcial y sesgadamente en las políticas públicas y menos aún en las prácticas institucionales concretas, y en la mayoría de los casos tampoco lo hacen en las políticas y prácticas de las universidades y otras IES "convencionales" (MATO, 2012).

En el marco de esas historias largas, del desarrollo de diversos factores internacionales, y de las luchas de los pueblos indígenas y de diversos acto- 
res sociales no-indígenas, especialmente en las últimas dos décadas, algunas organizaciones indígenas han creado programas, universidades y otras IES, propias o mediante alianzas con universidades y otras instituciones de educación superior convencionales. En tanto, algunos Estados, universidades y otras IES convencionales, fundaciones privadas y agencias de cooperación internacional han creado otras, y también han establecido algunas políticas y programas especiales. Todo esto ha dado lugar a un panorama bastante diverso de experiencias de colaboración en materia de educación superior entre pueblos indígenas, universidades y otras IES y otros actores, con sus logros, problemas, desafíos y conflictos que describiré sintéticamente en las próximas páginas.

\section{Modalidades de colaboración entre universidades, IES y pueblos indígenas, e iniciativas propias de organizaciones indígenas}

El panorama que se presenta en estas páginas se basa principalmente en las investigaciones realizadas por el Proyecto Diversidad Cultural e Interculturalidad en Educación Superior (en adelante, el o nuestro Proyecto) del Instituto Internacional de la UNESCO para la Educación Superior en América Latina y el Caribe (UNESCO-IESALC), el cual se me encargó crear en 2007, y coordino desde entonces ${ }^{3}$. Esta investigación no es exhaustiva y las experiencias que mencionaré constituyen sólo algunos ejemplos de las modalidades de colaboración que hemos logrado identificar. Este es un universo constituido por experiencias muy diferentes entre sí. Esta diversidad de experiencias no

\footnotetext{
${ }^{3}$ Además de la publicación de los mencionados cuatro libros, el desarrollo del Proyecto ha conducido a crear el Observatorio de Diversidad Cultural e Interculturalidad en Educación Superior, así como a ofrecer un Curso a Distancia de Formadores en Educación Superior Indígena y Afrodescendiente, el cual contó 50 participantes de 16 países, y también a realizar un concurso de fondos mediante el cual se apoyaron 8 proyectos de investigación y promoción del buen vivir, los cuales fueron seleccionados entre 210 postulaciones. Además, dio lugar a la creación de un Foro virtual de intercambio de experiencias para analizar el impacto de la formación de maestros y profesores afrodescendientes e indígenas en instituciones interculturales y diversas de Educación Superior en América Latina y el Caribe, que contó con 159 participantes de 11 países. Asimismo, se ha creado una Biblioteca Virtual temática y un Directorio de Instituciones vinculadas con actividades en este campo. Finalmente, en mayo de 2012 se realizó un Taller Regional sobre Políticas Públicas de Educación Superior y Pueblos Indígenas y Afrodescendientes en América Latina, en la Universidad de Panamá, con la participación de 31 especialistas provenientes de 12 países latinoamericanos, el cual dio como resultado la conceptualización y lanzamiento de la Iniciativa Latinoamericana por la Diversidad Cultural y la Interculturalidad con Equidad en Educación Superior y de su anexo de Propuestas de Acción, contentiva de una serie de recomendaciones orientadas a lograr que la Educación Superior sea más pertinente con la diversidad cultural propia de los distintos países de la región. Para más información sobre el Proyecto Diversidad Cultural e Interculturalidad en Educación Superior en América Latina, del Instituto Internacional de la UNESCO para la Educación Superior en América Latina y el Caribe (UNESCO-IESALC), ver: <http:/ / www.iesalc.unesco.org.ve/index. php?option=com_content\&view=article\&id=22\&Itemid=405\&lang=es $>$.
}

Tellus, ano 13, n. 25, jul./dez. 2013 
debería resultar sorprendente si tomamos en cuenta que se trata de modalidades de colaboración intercultural en las que participan comunidades y organizaciones de pueblos indígenas muy diferentes entre sí, en sociedades nacionales también muy distintas unas de otras.

A manera de síntesis, cabe adelantar que algunas de las modalidades de colaboración existentes orientan su accionar principalmente a asegurar la formación de profesionales y técnicos indígenas a través de su inclusión en universidades y otros tipos de IES convencionales. Otras procuran lograrla en el marco de universidades que se autoidentifican como "interculturales", o de programas especiales de universidades "convencionales", cuyos planes de estudio integran las lenguas y conocimientos propios de estos pueblos con las ciencias y humanidades "occidentales modernas". Otras modalidades son resultado de alianzas entre organizaciones indígenas y universidades y otras IES. Finalmente, otras modalidades son iniciativas propias de organizaciones indígenas, gestionadas por ellas mismas, cuyos planteles frecuentemente incluyen también docentes no-indígenas, generalmente llamadas "universidades indígenas", si bien existen algunos casos en los cuales no utilizan la denominación de "universidad". Podemos clasificar las experiencias de colaboración intercultural que hasta ahora ha logrado identificar nuestro Proyecto en cinco modalidades diferentes:

2.1 Programas de "inclusión de individuos" indígenas como estudiantes en instituciones de educación superior "convencionales"

Los programas de becas y cupos especiales para personas indígenas en universidades u otras instituciones de educación superior "convencionales" constituyen modalidades de "inclusión de individuos". Otra modalidad "de inclusión de individuos" es la de los programas de apoyo académico y psicosocial a estudiantes de esos pueblos. Las modalidades de "inclusión de individuos" no han formado parte de los focos de estudio del Proyecto, no obstante hemos tomado en cuenta algunas y el relevamiento realizado permite afirmar que existen numerosas experiencias que responden a estos tres tipos de modalidades (cupos, becas, y programas de apoyo), así como que, pese a su número, aún resultan insuficientes en comparación con las necesidades y demandas expresadas al respecto. Varios Estados y universidades públicas y privadas de América Latina han establecido programas de cupos y de becas, otro tanto han hecho varias fundaciones privadas. La Fundación Ford ha jugado un importante papel en la materia, sosteniendo dos importantes programas de este tipo; uno de becas de posgrado, el International Fellowships Program, y otro de apoyo académico y psicosocial que también procuraba lograr mejoras institucionales al respecto en algunas universidades, el Pathways 
to Higher Education (DIDOU AUPETIT; REMEDI ALLIONE, 2008; SOUZA LIMA; PALADINO, 2012; NAVARRETE, 2011).

Existen opiniones encontradas respecto de las experiencias de "inclusión de individuos". Algunas enfatizan que no sólo crean oportunidades para individuos, sino que además contribuyen a desarrollar capas de profesionales indígenas que sirven de manera directa a sus pueblos y comunidades, y que además muchos de estos profesionales juegan papeles fundamentales en el desarrollo y gestión de experiencias de los cuatro tipos restantes de esta clasificación. Otras opiniones destacan que este tipo de programas favorecen la "fuga de cerebros" desde las comunidades apartadas hacia las grandes ciudades, así como su "occidentalización", la pérdida de sus valores étnicos, de sus lenguas y de la valoración por sus saberes y el debilitamiento de sus vínculos con sus comunidades de origen. Otras señalan que con las becas no basta, porque en las universidades "convencionales" estos estudiantes frecuentemente deben lidiar con problemas de racismo, además de que en la curricula no encuentran reflejadas las historias, idiomas y conocimientos de sus pueblos, a lo que se añade que se encuentran alejados de sus familias y comunidades, y así de sus redes sociales de apoyo. Un detalle de interés es que varios de los profesionales indígenas que han sido autores de capítulos de los libros publicados por nuestro Proyecto han logrado realizar estudios universitarios gracias precisamente a programas de inclusión de individuos. Otro detalle de interés, es que si bien no manejo estadísticas al respecto, en mis visitas a universidades interculturales e indígenas en varios países latinoamericanos he conocido un buen número de docentes indígenas quienes también lograron realizar estudios universitarios gracias a estos tipos de programas.

No obstante, por no haber formado parte de los focos de nuestro estudio y existir tantas y tan diversas experiencias de programas de "inclusión de individuos" nuestro Proyecto no posee conocimientos suficientes para sacar sus propias conclusiones sobre este tipo de modalidades de colaboración intercultural.

2.2 Colaboración intercultural en programas de formación, conducentes a títulos u otras certificaciones, creados por universidades y otras IES "convencionales"

Existen diversas modalidades dentro de este grupo de experiencias. En algunas de ellas se registra escasa colaboración intercultural, mientras que en otras la participación de organizaciones y comunidades indígenas (o de ser el caso afrodescendientes) resulta significativa. En algunos de estos casos se constata además una participación importante de docentes provenientes de los mencionados pueblos y la inclusión de sus lenguas, conocimientos, 
formas de aprendizaje y modos de producción de conocimiento. Muchas de estas experiencias están orientadas a la formación de docentes para programas de educación intercultural bilingüe a diversos niveles del sistema educativo. Esta es otra modalidad de colaboración intercultural de la existe un número importante de experiencias.

Entre las experiencias de este tipo que han sido documentadas por nuestro Proyecto, es posible mencionar, por ejemplo, el Programa Académico Cotopaxi de la Universidad Politécnica Salesiana, en Ecuador (FARFÁN, 2008), varias experiencias desarrolladas por la Universidad de Cuenca, también en Ecuador (MENDOZA ORELLANA, 2008), la Licenciatura en Etnoeducación de la Universidad del Cauca, en Colombia (ROJAS, 2008), el Programa de Técnicos Superiores en Justicia Comunitaria, de la Universidad Mayor San Andrés, en Bolivia (MALLEA RADA, 2008) y el Programa de Formación en Educación Intercultural Bilingüe para los países andinos, de la Universidad Mayor San Simón, también en Bolivia (LIMACHI PÉREZ, 2008), así como las Licenciaturas para la formación de Profesores Indígenas de la Universidad do Estado de Mato Grosso y de la Universidad Federal de Roraima, en Brasil (CARVALHO; CARVALHO, 2008; JANUARIO; SELLERI SILVA, 2008).

Por otra parte, el Registro de Experiencias creado por nuestro Proyecto, permitió identificar varias experiencias de programas de formación conducentes a títulos $u$ otras certificaciones que no hemos estudiado pero cuya existencia debe cuanto menos mencionarse. Por ejemplo, cabe mencionar que en Brasil tenemos información de que existen además otras dieciocho licenciaturas pedagógicas además de las dos antes nombradas (OLIVEIRAPANKARARU, 2012, p. 46) ${ }^{4}$. En tanto, en Ecuador, desde 1992 la Universidad Estatal de Bolívar, una universidad pública, ha ofrecido licenciaturas en campos tales como Educación y Estudios Interculturales, Desarrollo Regional Intercultural, entre otros. En esta experiencia, de manera análoga a lo que ocurre en la de la Licenciatura en Desarrollo Comunitario Integral de la Universidad Pedagógica Nacional, una universidad pública de México, no sólo participan personas indígenas como estudiantes, sino que a través de diversas modalidades se incorporan los conocimientos de los sabios y ancianos de las comunidades. Otro tanto cabe decir de la experiencia de la especialidad de

\footnotetext{
${ }^{4}$ Las 20 universidades públicas brasileras que ofrecen cursos dedicados a la formación de docentes indígenas, que en este país reciben el nombre de Licenciatura Intercultural, son: Universidade Federal do Acre, Universidade Federal do Amazonas, Instituto Federal de Educação, Ciência e Tecnologia do Amazonas, Universidade do Estado do Amazonas, Universidade Federal do Amapá, Universidade Federal de Rondônia, Universidade Federal de Roraima, Universidade do Estado de Alagoas, Instituto Federal da Bahia (Campus Porto Seguro) Universidade do Estado da Bahia, Universidade Federal do Ceará, Universidade do Estado do Ceará, Universidade Federal de Campina Grande, Universidade Federal de Pernambuco (Campus de Caruaru), Universidade Federal de Minas Gerais, Universidade Federal de Santa Catarina, Universidade Federal de Goiás, Universidade Federal de Mato Grosso do Sul, Universidade Federal da Grande Dourados, Universidade do Estado do Mato Grosso (OLIVEIRA-PANKARARU, 2012).
} 
Educación Intercultural Bilingüe para la formación de maestros de educación básica y secundaria ofrecida en la Universidad Nacional de Educación Enrique Guzmán y Valle La Cantuta, una universidad pública peruana.

En muchas de las experiencias de este tipo se registra la participación de sabios de las comunidades indígenas cercanas en calidad de docentes. Junto con esto se presenta el problema de que por no contar con título universitario (y en ocasiones tampoco de otros niveles educativos) esto sabios no son propiamente reconocidos como docentes, ni remunerados como tales. Sin embargo, en general son los únicos y sino, como mínimo, quienes mejor pueden dar clases de idiomas indígenas, o sobre las respectivas historias y cosmovisiones. En ocasiones trabajan a través de la modalidad llamada de "pareja-pedagógica", en cuyo marco suelen ser colocados en situación subalterna, y frecuentemente reciben menores remuneraciones $u$ beneficios laborales. Estas situaciones además de ser injustas constituyen nuevos mecanismos de subalternización de los conocimientos, las lenguas y las personas indígenas.

Un problema que suelen confrontar muchos de los programas especiales de este tipo es que no cuentan con financiamiento regular de las universidades, ni del presupuesto nacional o estadual de los respectivos países, sino que dependen de convenios con agencias de cooperación, o convocatorias de fondos especiales de los respectivos gobiernos. Esto, obviamente genera problemas de sostenibilidad. Muchos de estos programas están dirigidos a docentes en servicio en comunidades indígenas. Aprovechando esta posibilidad, suelen combinar modalidades de enseñanza en las aulas de los campus universitarios con formas de enseñanza/aprendizaje en servicio en las escuelas de las comunidades en las cuales estos estudiantes universitarios son docentes. Esta modalidad de trabajo, que por lo general ha demostrado ser muy provechosa, en ocasiones ha sido cuestionada, e incluso no reconocida por las agencias estatales de algunos países encargadas de certificar la calidad académica de los programas universitarios.

2.3 Colaboración intercultural en proyectos de docencia, investigación, y/o vinculación social, desarrollados por universidades y otras IES "convencionales" con participación de comunidades de pueblos indígenas

El conjunto de experiencias que un tanto forzadamente incluyo en esta modalidad es expresión de la diversidad de contextos, culturas profesionales y académicas, culturas institucionales, y normativas monoculturalistas, que ponen a prueba la creatividad de numerosos grupos de docentes, investigadores y otros actores significativos. Por esto, bajo la complicada denominación de esta modalidad he agrupado un conjunto diverso de experiencias, que un tanto 
arbitrariamente se podrían separar en tres subconjuntos, pero el problema es que algunas formarían parte de más de uno de éstos.

El primero de esos subconjuntos abarcaría las experiencias centradas principalmente en programas o proyectos de docencia, algunos de los cuales incluyen componentes de investigación y/o de vinculación con la comunidad. Se diferencian de los del tipo descrito en la sección anterior (2.2) en que no conducen a títulos de grado o postgrado, sino que consisten en una o más asignaturas o seminarios, que no otorgan créditos, puntos, u horas para graduarse. Dependiendo de los casos, este tipo de actividades se caracteriza por incluir la participación de docentes provenientes de los pueblos indígenas y la inclusión significativa de lenguas, saberes y modos de producción de conocimiento y aprendizaje propios de estos pueblos.

El segundo subconjunto abarcaría experiencias en las cuales la colaboración intercultural se da principalmente a través de programas o proyectos de investigación, algunos de los cuales además incluyen actividades docentes, otras no. Según los casos estas experiencias producen conocimientos sobre estas comunidades, sistematizan sus idiomas y conocimientos, o generan tecnologías, que comparten de manera pro-activa con las comunidades, o bien hacen esto mismo pero mediante modalidades co-trabajo o coproducción.

El tercer subgrupo incluiría experiencias en las que la colaboración se da especialmente mediante proyectos denominados de "vinculación" o "servicio social" u otras denominaciones, orientados a mejorar la calidad de vida de las comunidades. No se limitan a "aplicar" saberes académicos en las comunidades, sino que integran saberes de las comunidades. Este tipo de casos se caracterizaría porque aunque incluyen actividades docentes y de investigación, sus actividades principales son de servicio a y vinculación con las comunidades.

En estos tres subtipos de experiencias suele observarse una importante participación de miembros de las comunidades con sus idiomas, conocimientos, y modos de organización de las actividades.

Existe un número indeterminado, pero seguramente muy grande, de experiencias de estos tipos ${ }^{5}$. El Registro de Experiencias creado por nuestro

\footnotetext{
${ }^{5}$ La afirmación de que este número habría de ser muy grande se basa no solo en la información recogida a través del registro antes mencionado y del conocimiento directo de otras no incluidas en dicho registro. También se basa en que en el período de poco menos de un mes (abril 2011) transcurrido entre el lanzamiento y cierre de la convocatoria realizada por el mencionado Proyecto de UNESCO-IESALC, invitando a participar en un concurso a través del cual se otorgarían solo diez fondos para financiar proyectos de investigación y promoción del buen vivir, desarrollados por instituciones de Educación Superior en colaboración con comunidades de pueblos indígenas y/o afrodescendientes, se recibieron 210 valiosos proyectos, presentados por instituciones de educación superior de 17 países de América Latina, que en la mayoría de los casos tenían relaciones de colaboración preexistentes con las comunidades en cuestión. No obstante, tal como preveían las bases del concurso, el jurado, constituido por cinco reconocidos especialistas en el tema, debió limitarse a seleccionar sólo diez proyectos. Si bien ese concurso
} 
Proyecto en 2009 ha permitido reunir información básica sobre cerca de cincuenta experiencias de los tres tipos antes mencionados, aunque solo hemos estudiado cinco de ellas en particular. Por limitaciones de extensión en este momento mencionaré solo dos de ellas. Una de estas ha sido el Programa Docencia e Investigación en Medicina Tradicional del Instituto Politécnico Nacional, una institución pública de educación superior de México, que ha integrado la participación de un equipo interdisciplinario de esa institución con la de profesores y niños de escuelas básicas, médicos tradicionales y campesinos del estado de Oaxaca (CAMACHO MORFIN et al., 2009). Otra ha sido el Programa de Investigación Interdisciplinario Desarrollo Humano, iniciado en el año 1997, en el estado de Chiapas, por la Universidad Autónoma Metropolitana, una universidad pública mexicana. Este programa integra el trabajo de investigación con la formación profesional y el servicio a la comunidad. En este programa participan profesores, investigadores y estudiantes de diferentes departamentos, junto a miembros de las comunidades (CORTEZ RUIZ, 2009).

2.4 Colaboración intercultural entre universidades u otras IES y organizaciones indígenas, en co-ejecuciones orientadas a responder a propuestas de formación de estas últimas

Hasta el momento hemos logrado identificar sólo cuatro experiencias de este tipo, todas las cuales han sido documentadas en los libros de nuestro Proyecto. Todas ellas otorgan títulos reconocidos por las autoridades educativas nacionales de los respectivos países.

Dos de estas experiencias son sendas co-ejecuciones impulsadas por la Asociación Interétnica de Desarrollo de la Selva Peruana (AIDESEP). Una de éstas es el Programa de Formación de Maestros Bilingües de la Amazonía Peruana, que desarrolla en colaboración con el Instituto Superior Pedagógico de Loreto (TRAPNELL, 2008) y la otra el Programa de Formación de Enfermeros Técnicos en Salud Intercultural, que desarrolla en colaboración con

estaba abierto también a proyectos con comunidades afrodescendientes, una muy significativa mayoría de los proyectos recibidos estaba orientada a trabajar con comunidades de pueblos indígenas. Esto constituye un indicador de que existe un universo bastante mayor de experiencias de este tipo. Resulta interesante destacar que dado que las bases del concurso incluían la condición de que los proyectos debían incluir "la participación y conocimientos de dichas comunidades", prácticamente la totalidad de los proyectos recibidos proponían, y sostenían haber desarrollado con anterioridad, diversas formas de colaboración en la producción y/o aplicación de conocimientos académicos y los propios de las comunidades. Un detalle especialmente significativo es que, en algunos casos, entre los conocimientos de las comunidades se incluían no solo saberes ancestrales, sino otros relacionados con problemas contemporáneos de las mismas, frecuentemente referidos a asuntos de derechos humanos, incluyendo políticos y territoriales, educación, modelo de desarrollo local en el marco de los respectivos modelos nacionales hegemónicos, entre otros. 
el Instituto Superior Tecnológico Público de Atalaya, situado en la pequeña ciudad amazónica de este mismo nombre (RODRÍGUEZ TORRES et al., 2009).

Otras dos experiencias han sido desarrolladas por la Organización Indígena de Antioquia (OIC), de Colombia, a través de su Instituto de Educación Indígena, una de ellas en alianza con la Universidad Pontificia Bolivariana, y la otra con la Universidad de Antioquia, mediante las cuales ofrecen la Licenciatura en Etnoeducación, la especialización en Gobierno y Administración Indígena y la Licenciatura en Pedagogía de la Madre Tierra (CÁISAMO ISARAMA; GARCÍA, 2008; SIERRA, 2004).

Aunque hasta la fecha no hemos logrado identificar otras experiencias de este tipo, las estudiadas llevan a pensar que se trata de una modalidad potencialmente muy provechosa para la formación de profesionales y técnicos de los pueblos indígenas. Hemos obtenido información acerca de varias iniciativas de este tipo que no han logrado prosperar debido a prejuicios de ambas partes, así como a obstáculos burocráticos de las universidades, y otros asociados a las normativas de educación superior.

2.5 Experiencias de colaboración intercultural en Instituciones Interculturales de Educación Superior

Las universidades $\mathrm{u}$ otros tipos de Instituciones Interculturales de Educación Superior (IIES) se caracterizan por integrar los saberes, modos de producción de conocimiento y modos de aprendizaje de varias tradiciones culturales. En América Latina, las universidades u otros tipos de IES de este tipo, integran conocimientos tradicionales de uno o más pueblos indígenas con los correspondientes a la tradición occidental moderna, usualmente referidos como "ciencia" y "humanidades". Debe destacarse que, más allá de esta suerte de conceptualización - a grandes rasgos - común, las universidades y otras IES interculturales son muy diversas entre si, como consecuencia de las diferencias existentes entre diversos pueblos indígenas, las diversas historias de los Estados nacionales, diversos actores que han intervenido en su creación y otros factores. No existe un modelo de referencia, y dadas las diferencias apuntadas tampoco sería pertinente pretender establecerlo.

Como consecuencia del proceso histórico de colonización y las continuidades del mismo que pueden observarse en las repúblicas creadas a partir del siglo XIX, en América Latina, estas instituciones han sido creadas especialmente para atender las demandas y propuestas de formación de pueblos indígenas. Estas instituciones reciben principalmente estudiantes indígenas y en menor medida estudiantes no-indígenas. Desde hace tiempo, numerosos dirigentes y organizaciones indígenas, así como otros actores sociales, veni- 
mos insistiendo en que se debe "interculturalizar toda la educación superior". Argumentando en que, dada la condición pluricultural de los países latinoamericanos, todas las universidades y otros tipos de IES deberían ser interculturales (MATO, 2008b, 2012).

No obstante en vista del rezago de los Estados latinoamericanos en esta materia, dirigentes y organizaciones indígenas de varios países de la región se han dado a la tarea de crear instituciones propias. Nuestro proyecto ha documentado la experiencia de varias universidades de este tipo, como la Universidad Intercultural Indígena Originaria Kawsay, creada por una red de organizaciones indígenas de Bolivia, Perú y Ecuador (CERRUTO, 2009; SAAVEDRA, 2008); el Centro Amazónico de Formación Indígena, creado por la Coordinación de Organizaciones Indígenas de la Amazonía Brasileira, en Brasil (FLORES, 2009); la Universidad Autónoma, Indígena e Intercultural (UAIIN), creada por el Consejo Regional Indígena del Cauca (CRIC), en Colombia (BOLAÑOS et al., 2009); la Universidad Intercultural de las Nacionalidades y Pueblos Indígenas "Amawtay Wasi" (UINPI), creada por algunos sectores de la Confederación de Nacionalidades Indígenas del Ecuador (SARANGO, 2009); la Universidad de las Regiones Autónomas de la Costa Caribe Nicaragüense-URACCAN (HOOKER, 2009); la Bluefields Indian \& Caribbean University (CHAVARRÍA LEZAMA, 2008), estas dos últimas creadas en Nicaragua por iniciativas de liderazgos indígenas y afrodescendientes locales. Más recientemente hemos identificado otras instituciones de educación superior indígenas que aún no hemos estudiado, como la Universidad Intercultural de los Pueblos del Sur (Unisur), creada por un grupo de dirigentes indígenas del Estado de Guerrero, México, con apoyo de algunos académicos de otras universidades mexicanas y el Instituto de Educación Superior Intercultural "Campinta Guazú Gloria Pérez", creado por Consejo de Organizaciones Aborígenes de Jujuy, en Argentina.

Por otra parte, también existen universidades y otros tipos de IES de carácter intercultural que han sido creadas por organismos estatales de distinto nivel. Un ejemplo de esto es el Centro de Investigación y Formación para la Modalidad Aborigen, creado por el gobierno de la provincia del Chaco en Argentina (VALENZUELA, 2009), o bien los nueve institutos creados dependientes de la Dirección General de Educación Superior de la Provincia de Salta. Otro ejemplo de este tipo de instituciones es el sistema de las ya once universidades interculturales integrantes del sistema de universidades interculturales creado por la Secretaria de Educación Pública de México (CASILLAS; SANTINI, 2006; SCHMELKES, 2008), que es el organismo federal/nacional de este país, como las Universidades Interculturales de los Estados de Chiapas (FÁBREGAS PUIG, 2009), Guerrero, México, Puebla y Tabasco, así como la Universidad Intercultural Indígena de Michoacán y la 
Universidad Intercultural Maya de Quintana Roo; y también otras dos creadas inicialmente por gobiernos de dos estados de la federación (nivel provincial/ estadual) y posteriormente asimiladas al sistema nacional, la Universidad Autónoma Indígena de México (GUERRA GARCÍA; MEZA HERNÁNDEZ, 2009) y la Universidad Comunitaria de San Luis Potosí (SILVA CARRILLO, 2009), y la Universidad Veracruzana Intercultural, creada por la Universidad Veracruzana (DIETZ, 2008). En tanto, en Bolivia, en agosto de 2008, mediante un decreto presidencial se crearon tres Universidades Indígenas Comunitarias Interculturales de Bolivia (UNIBOL), la Universidad Indígena Aymara, la Universidad Quechua y la Universidad Guaraní y de Pueblos Indígenas de Tierras Bajas (CHOQUE QUISPE, 2012). En el año 2000 se creó en Perú la Universidad Nacional Intercultural de la Amazonía, que cesó actividades en 2010 y actualmente está en vías de reiniciarlas. En este mismo país, más recientemente se ha dispuesto la creación de otras tres universidades nacionales interculturales, la de Quillabamba, la de Bagua y la de la Selva Central, pero su funcionamiento aún confronta restricciones presupuestarias. En el caso de Argentina, en el año en curso, ante la Comisión de Educación de la Cámara de Diputados, se presentó un Proyecto de Ley para la Creación de la Universidad Nacional Intercultural de los Pueblos Indígenas (Expediente Nro. 5143-D-2013), a propósito del cual en agosto de 2013 se ha realizado una primera Audiencia Pública, pero hasta ahora la Comisión de Educación de esa Cámara no ha tratado el proyecto.

Existen además otros dos tipos de universidades interculturales. Por un lado, tenemos el caso de la Universidad Indígena Intercultural, que fue creada por el Fondo para el Desarrollo de los Pueblos Indígenas de América Latina y el Caribe, un organismo multilateral co-gobernado por un cuerpo de representantes de gobiernos y organizaciones indígenas (YAPU, 2008) que funciona principalmente a través de la Red de Universidades Indígenas Interculturales de Abaya Yala (entre las cuales están las ya nombradas URACCAN, UAIN, UINPI y las tres universidades del sistema UNIBOL). Por otro lado, tenemos el caso de la Universidad Intercultural Ayuk, creada en México como parte del sistema de la Universidad Iberoamericana, de los jesuitas (ESTRADA, 2008).

\section{Logros, problemas, desafíos y conflictos}

Desde 2007, el Proyecto Diversidad Cultural e Interculturalidad en Educación Superior de UNESCO-IESALC, gracias a la colaboración de casi setenta investigadores de once países latinoamericanos, ha realizado estudios específicos sobre aproximadamente cuarenta experiencias de los tipos antes mencionados, así como sobre los respectivos contextos. Estos estudios han sido incluidos en cuatro libros que están disponibles tanto en versión impresa 
como en versión digital en Internet (MATO, 2008b; 2009a; 2009b; 2012). Esos estudios entre otras cosas han permitido identificar los logros, problemas, y desafíos más salientes de esas experiencias, así como algunas áreas de conflicto.

Logros: Los principales logros de los programas, universidades y otras IES estudiadas son: i) mejoran las posibilidades de que individuos indígenas y afrodescendientes accedan a oportunidades de educación superior y culminen exitosamente sus estudios, ii) ajustan su oferta educativa a necesidades, demandas y proyectos de las comunidades y la relacionan con oportunidades locales y regionales (subnacionales) de empleo, generación de iniciativas productivas y servicio a la comunidad, iii) desarrollan modalidades participativas de aprendizaje centradas en la investigación aplicada, iv) integran docencia/aprendizaje, investigación y servicio a las comunidades, v) integran diversos tipos de saberes y modos de producción de conocimiento, vi) promueven la valorización y, según los casos, incorporan lenguas y conocimientos propios de estos pueblos y comunidades, contribuyen proactivamente a su fortalecimiento y realizan investigación sobre ellos, vii) desarrollan docencia e investigación orientados por criterios de valoración de la diversidad cultural, interculturalidad, equidad, inclusión, gobernabilidad democrática, desarrollo humano y sostenible, viii) forman egresados que contribuyen al desarrollo sostenible local y regional y al mejoramiento de la calidad de vida de sus comunidades.

Problemas y desafíos: Los problemas y desafíos más frecuentemente enfrentados por estas Universidades, Institutos y programas son: i) insuficiencia y/o precariedad presupuestaria, ii) actitudes racistas por parte de funcionarios públicos y diversos sectores de población que afectan el desarrollo de sus actividades, iii) dificultades derivadas de la rigidez de los criterios de evaluación aplicados por las agencias especializadas de los Estados encargadas de otorgar reconocimiento y/o acreditación, iv) obstáculos institucionales derivados de la rigidez de procedimientos administrativos, los cuales afectan la ejecución de sus planes y actividades, v) obstáculos institucionales derivados de la rigidez de criterios académicos aplicados por las IES dentro de las cuales funcionan algunos de los programas estudiados, y/o por agencias gubernamentales que otorgan fondos para investigación y proyectos especiales, los cuales afectan labores docentes y de investigación de las IES y programas estudiados, y muy particularmente dificultan la posibilidad de sumar las contribuciones de sabias/os indígenas, vi) dificultades para conseguir docentes y otro personal con adecuada sensibilidad y recursos personales y técnicos para el trabajo intercultural, vii) dificultades económicas de los estudiantes para poder dedicarse más y mejor a su formación, viii) insuficiencia de becas.

Conflictos: Buena parte de los conflictos que se presentan entre estos tipos de universidades e institutos y los respectivos Estados nacionales giran 
en torno a los mecanismos y criterios de evaluación y reconocimiento aplicados por las agencias de los Estados encargadas de estos asuntos. La evaluación de la calidad de las instituciones y programas de educación (superior o de otro tipo) no puede disociarse de consideraciones de pertinencia y relevancia, esto demanda que sea contextualizada. No resulta apropiado aplicar sistemas rígidos de criterios de evaluación de validez supuestamente "universal". Es necesario incorporar criterios que, partiendo de reconocer la heterogeneidad de cada sociedad, respondan a las diferencias, para asegurar la pertinencia de las instituciones y programas de educación (superior o de otro tipo) con la diversidad de sus contextos sociales.

\section{Conclusiones}

La evaluación de los programas e instituciones interculturales de educación superior, como la de sus docentes e investigadores, requiere diseñar instrumentos que tomen en cuenta la especificidad de las propuestas y de los contextos en que se desarrollan. Es necesario revisar los criterios de evaluación y acreditación de las IES y programas, así como los aplicados para la asignación de fondos para investigación y proyectos; para asegurar que éstos respondan a criterios de reconocimiento y valoración de la diversidad cultural y la interculturalidad con equidad. Estos criterios deben responder a las peculiaridades de los diversos tipos de IES, con especial atención a diferencias asociadas a particularidades lingüísticas, socioculturales, territoriales y otras significativas, según la diversidad cultural propia de cada país.

Además, y muy importante, es necesario cambiar radicalmente los estilos de evaluación de los programas, universidades y otros tipos de IES interculturales y/o indígenas. Estos suelen ser procesos de carácter punitivo que las agencias de evaluación y acreditación de los Estados emprenden unilateralmente, aplicando instrumentos propios, no consensuados. No son procesos interculturales, y en este sentido no se ajustan a lo dispuesto por el Convenio 169 de la OIT que establece el carácter obligatorio de la consulta previa a los pueblos indígenas en todos los asuntos que les conciernen. Además, tampoco respetan lo establecido el acápite C3 de la Declaración de la Conferencia Regional de Educación Superior, suscrita por las autoridades educativas y universitarias de la región, que establece que "el reto no es sólo incluir a indígenas, afrodescendientes y otras personas culturalmente diferenciadas en las instituciones tal cual existen en la actualidad, sino transformar a éstas para que sean más pertinentes con la diversidad cultural". Ese mismo acápite, también dictamina la necesidad de "incorporar el diálogo de saberes y el reconocimiento de la diversidad de valores y modos de aprendizaje como elementos centrales de las políticas, planes y programas del sector". 
Es necesario poner en práctica modalidades de diseño, evaluación y acreditación de planes de estudio y de universidades y otros tipos de IES que sean verdaderamente interculturales. Esto quiere decir que se caractericen por asegurar la participación informada y activa de las respectivas instituciones, así como de las comunidades y organizaciones indígenas, y muy especialmente que sean de proceso. Es decir, orientadas a construir formas mutuamente respetuosas y equitativas de diálogo y colaboración intercultural tendientes a mejorar las experiencias no a simplemente examinarlas unilateralmente y asignarles una calificación. Para esto es necesario que los Estados asignen los "recursos apropiados", tal como lo establece el artículo 27 del Convenio 169 de la OIT que ha sido ratificado por 14 Estados latinoamericanos, entre otros el Estado argentino.

Junto con los cambios hasta acá mencionados, también es necesario revisar críticamente ciertas ideas transnacionalizadas de "calidad académica" que crecientemente han venido adquiriendo carácter de "sentido común" en los medios masivos de comunicación y las agendas gubernamentales. Estas ideas valoran la medición de la llamada "calidad académica" preponderantemente en términos de publicaciones en revistas especializadas y otros indicadores ciencio-métricos, pero no valoran la vinculación de las universidades con los respectivos contextos, y en qué medida responden a sus necesidades, demandas y propuestas. De este modo, afectan las posibilidades de desarrollo de orientaciones y modalidades de trabajo propias por parte de todas las universidades latinoamericanas mundo (no solo de las interculturales), tendiendo a encorsetarlas dentro de modelos universitarios propios de otras otros tipos de contextos sociales, en otras regiones (MATO, 2013). Es necesario revisar críticamente esas ideas transnacionalizadas de "calidad académica" y los sistemas de indicadores asociados, para pasar a utilizar indicadores integrados de calidad, pertinencia, y relevancia, en la evaluación de todas las universidades y otros tipos de IES.

\section{Referencias}

BOLAÑOS, Graciela; TATAY, Libia; PANCHO, Avelina. Universidad Autónoma, Indígena e Intercultural (UAIIN): un proceso para fortalecer la educación propia y comunitaria en el marco de la interculturalidad. En: MATO, Daniel (Coord.). Instituciones Interculturales de Educación Superior en América Latina. Procesos de construcción, Logros, Innovaciones y Desafíos. Caracas: UNESCO-IESALC, 2009. p. 155-190.

CÁISAMO ISARAMA, Guzmán; GARCÍA CASTRO, Laura. Experiencias en educación superior de la Organización Indígena de Antioquia y su Instituto de Educación Indígena en alianza con la Universidad Pontificia Bolivariana y la Universidad de Antioquia. En: MATO, Daniel (Coord.). Diversidad cultural e interculturalidad en 
educación superior. Experiencias en América Latina. Caracas: UNESCO-IESALC, 2008. p. 223-232.

CAMACHO MORFIN, Roxana; GUZMÁN FÉLIX, Cándido E.; DÍAZ HERNÁNDEZ, Joaquín. Una Experiencia Intercultural a partir del Conocimiento Etnomédico. En: MATO, Daniel (Coord.). Educación superior, colaboración intercultural y Desarrollo Sostenible/Buen Vivir. Experiencias en América Latina. Caracas: IESALC-UNESCO, 2009. p. 65-86.

CARVALHO, Fabíola; CARVALHO, Fabio Almeida de. A experiência de formação de professores indígenas do Núcleo Insikiran da Universidade Federal de Roraima (Brasil). En: MATO, Daniel (Coord.). Diversidad cultural e interculturalidad en educación superior. Experiencias en América Latina. Caracas: UNESCO-IESALC, 2008. p. 157-166.

CERRUTO, Leonel. La experiencia de la Universidad Indígena Intercultural Kawsay (UNIK). En: MATO, Daniel (Coord.). Instituciones Interculturales de Educación Superior en América Latina. Procesos de construcción, logros, innovaciones y desafíos. Caracas: IESALC-UNESCO, 2009. p. 123-153.

CHAVARRÍA LEZAMA, Pedro. La interculturalidad: un desafío ineludible de la Bluefields Indian \& Caribbean University. En: MATO, Daniel (Coord.). Diversidad cultural e interculturalidad en educación superior. Experiencias en América Latina. Caracas: UNESCO-IESALC, 2008. p. 393-401.

CHOQUE QUISPE, María Eugenia. Políticas de Educación para Pueblos Indígenas, originario, campesino y afro-descendientes en Bolivia. En: MATO, Daniel (Coord.). Educación Superior y Pueblos Indígenas y Afrodescendientes en América Latina. Normas, Políticas y Prácticas. Caracas: Instituto Internacional de la UNESCO para la Educación Superior en América Latina y el Caribe (IESALC-UNESCO), 2012.

CORTEZ RUIZ, Carlos. El Vínculo Universidad - Sociedad en la Experiencia del Programa de Investigación Interdisciplinario "Desarrollo Humano" de la Universidad Autónoma Metropolitana, México. En: MATO, Daniel (Coord.). Educación Superior, Colaboración Intercultural y Desarrollo Sostenible/Buen Vivir. Experiencias en América Latina. Caracas: IESALC-UNESCO, 2009. p. 115-142.

DIDOU AUPETIT, Sylvie; REMEDI ALLIONE, Eduardo. Pathways en América Latina: acción afirmativa de base étnica e instituciones de educación superior en América Latina. México DF: Fundación Ford y Centro de Investigación y de Estudios Avanzados del Instituto Politécnico Nacional, 2008.

DIETZ, Gunther. La experiencia de la Universidad Veracruzana Intercultural. En: MATO, Daniel (Coord.). Diversidad Cultural e Interculturalidad en Educación Superior. Experiencias en América Latina. Caracas: IESALC-UNESCO, 2008. p. 359-370.

ESTRADA, Guillermo. Sobre la experiencia del Centro de Estudios Ayuuk-Universidad Indígena Intercultural Ayuuk. En: MATO, Daniel (Coord.). Diversidad Cultural e Interculturalidad en Educación Superior. Experiencias en América Latina. Caracas: IESALC-UNESCO, 2008. p. 371-381. 
FÁBREGAS PUIG, Andrés. Cuatro años de Educación Superior Intercultural en Chiapas, México. En: MATO, Daniel (Coord.). Instituciones Interculturales de Educación Superior en América Latina. Procesos de Construcción, Logros, Innovaciones y Desafíos. Caracas: UNESCO-IESALC, 2009. p. 251-278.

FARFÁN, Marcelo. Experiencia del Programa Académico Cotopaxi, Formación en Educación Intercultural Bilingüe de la Universidad Politécnica Salesiana. En: MATO, Daniel (Coord.). Diversidad cultural e interculturalidad en Educación Superior. Experiencias en América Latina. Caracas: Instituto Internacional para la Educación Superior en América Latina y el Caribe (IESALC)-UNESCO, 2008. p. 285-296.

FLORES, Lucio. Centro Amazônico de Formação Indígena (CAFI), Coordenação das Organizações Indígenas da Amazônia Brasileira - COIAB. En: MATO, Daniel (Coord.). Instituciones Interculturales de Educación Superior en América Latina. Procesos de Construcción, Logros, Innovaciones y Desafíos. Caracas: UNESCO-IESALC, 2009. p. 79-102.

GUERRA GARCÍA, Ernesto; MEZA HERNÁNDEZ, María Eugenia. El corto y sinuoso camino de la Universidad Autónoma Indígena de México (UAIM). En: MATO, Daniel (Coord.). Instituciones Interculturales de Educación Superior en América Latina. Procesos de Construcción, Logros, Innovaciones y Desafíos. Caracas: UNESCO-IESALC, 2009. p. 215-250.

HOOKER BLANDFORD, Alta Suzzane. La Universidad de las Regiones Autónomas de la Costa Caribe Nicaragüense (URACCAN): Logros, innovaciones y desafíos. En: MATO, Daniel (Coord.). Instituciones Interculturales de Educación Superior en América Latina. Procesos de Construcción, Logros, Innovaciones y Desafíos. Caracas: UNESCO-IESALC, 2009. p. 279-301.

JANUARIO, Elias; SELLERISILVA, Fernado. Estudo sobre a experiência dos Cursos de Licenciatura para a Formação de Professores Indígenas da Universidade do Estado de Mato Grosso (Brasil). En: MATO, Daniel (Coord.). Diversidad cultural e interculturalidad em educación superior. Experiencias en América Latina. Caracas: UNESCO-IESALC, 2008. p. 147-156.

LIMACHI PÉREZ, Vicente. La experiencia del Programa de Formación en Educación Intercultural Bilingüe para los países andinos (PROEIB-Andes), sede Universidad Mayor de San Simón, Bolivia. En: MATO, Daniel (Coord.). Diversidad Cultural e Interculturalidad en Educación Superior. Experiencias en América Latina. Caracas: IESALC-UNESCO, 2008. p. 439-448.

MALLEA RADA, Julio A.). El Programa de Técnicos Superiores en Justicia Comunitaria de la Facultad de Derecho y Ciencias Políticas de la Universidad Mayor de San Andrés. En: MATO, Daniel (Coord.). Diversidad Cultural e Interculturalidad en Educación Superior. Experiencias en América Latina. Caracas: IESALC-UNESCO, 2008. p. 107-114.

MATO, Daniel. No hay saber "universal": la colaboración intercultural en la producción de conocimientos es imprescindible. Alteridades, Iztapalapa, México (Departamento de Antropología, Universidad Autónoma Metropolitana), 18(35): 101-116, 2008a. 
MATO, Daniel (Coord.). Diversidad cultural e interculturalidad en educación superior. Experiencias en América Latina. Caracas: Instituto Internacional de la UNESCO para la Educación Superior en América Latina y el Caribe (UNESCO-IESALC), 2008b.

MATO, Daniel. Contribución de experiencias de vinculación social de las universidades al mejoramiento de la calidad académica y factores que limitan su desarrollo y valoración institucional. Revista Avaliação: Revista da Avaliação da Educação Superior (Universidade de Sorocaba), Sao Paulo, Brasil, 18(1): 151-180, 2013.

MATO, Daniel (Coord.). Instituciones interculturales de educación superior en América Latina. Procesos de construcción, logros, innovaciones y desafíos. Caracas: Instituto Internacional de la UNESCO para la Educación Superior en América Latina y el Caribe (UNESCO-IESALC), 2009a.

MATO, Daniel (Coord.). Educación superior, colaboración intercultural y desarrollo sostenible/ buen vivir. Experiencias en América Latina. Caracas: Instituto Internacional de la UNESCO para la Educación Superior en América Latina y el Caribe (UNESCO-IESALC), 2009b.

MATO, Daniel (Coord.). Educación Superior y Pueblos Indígenas y Afrodescendientes en América Latina. Normas, políticas, y prácticas. Caracas: Instituto Internacional de la UNESCO para la Educación Superior en América Latina y el Caribe (UNESCO-IESALC), 2012.

MENDOZA ORELLANA, Alejandro. La Universidad de Cuenca: su compromiso con la formación universitaria de las nacionalidades indígenas del Ecuador. En: Daniel MATO, (Coord.). Diversidad cultural e interculturalidad en Educación Superior. Experiencias en América Latina. Caracas: Instituto Internacional para la Educación Superior en América Latina y el Caribe (IESALC), UNESCO, 2008. p. 275-284.

NAVARRETE, David. Becas, inclusión social y equidad en el prosgrado. Una aproximación desde el Programa Internacional de Becas para Indígenas. Perfiles Educativos, Coyoacán, v. 33, n.spe., p. 262-272, 2011.

OLIVEIRA-PANKARARU, Maria das Dores. As políticas públicas de Educação superior para indígenas e afrodescendentes no Brasil. Perspectivas e desafíos. En: MATO, Daniel (Coord.). Educación superior y pueblos indígenas y afrodescendientes en América Latina. Normas, políticas, y prácticas. Caracas: Instituto Internacional de la UNESCO para la Educación Superior en América Latina y el Caribe (UNESCO-IESALC), 2012. p. 139-176.

RODRÍGUEZ TORRES, Raúl et al. Formación de enfermeros técnicos en salud intercultural: una experiencia de cooperación en la Amazonía peruana. En: MATO, Daniel (Coord.). Educación Superior, colaboración intercultural y desarrollo sostenible/Buen Vivir. Experiencias en América Latina. Caracas: IESALC-UNESCO, 2009. p. 87-114.

ROJAS, Axel. ¿Etnoeducación o educación intercultural? Estudio de caso sobre la licenciatura en Etnoeducación de la Universidad del Cauca. En: Daniel MATO, coord. Diversidad cultural e interculturalidad em educación superior. Experiencias en América Latina. Caracas: UNESCO-IESALC, 2008. p. 233-242. 
SAAVEDRA, José Luis. Universidad Intercultural Indígena Originaria Kawsay. En: MATO, Daniel (Coord.). Diversidad cultural e interculturalidad em educación superior. Experiencias en América Latina. Caracas: UNESCO-IESALC, 2008. p. 115-124.

SARANGO, Luis Fernando. Universidad Intercultural de las Nacionalidades y Pueblos Indígenas "Amawtay Wasi". Ecuador / Chinchaysuyu. En Daniel MATO, coord. Instituciones interculturales de educación superior en América Latina. Procesos de Construcción, Logros, Innovaciones y Desafíos. Caracas: UNESCO-IESALC, 2009. p. 191-214.

SCHMELKES, Sylvia. Creación y desarrollo inicial de las universidades interculturales en México: problemas, oportunidades, retos. En: MATO, Daniel (Coord.). Diversidad Cultural e Interculturalidad en Educación Superior. Experiencias en América Latina. Caracas: IESALC-UNESCO, 2008. p. 329-338.

SIERRA, Zaida (Ed.). Voces indígenas universitarias. Expectativas, vivencias y sueños. Medellín: Universidad de Antioquia, 2004.

SILVA CARRILLO, Salvador. Universidad Comunitaria de San Luis Potosí. En: MATO, Daniel (Coord.). Educación superior, colaboración intercultural y desarrollo sostenible/Buen Vivir. Experiencias en América Latina. Caracas: IESALC-UNESCO, 2009. p. 163-182.

SOUZA LIMA, Antonio Carlos de; PALADINO, Mariana (Org.). Caminos hacia la educación superior: los programas Pathways de la Fundación Ford para pueblos indígenas en México, Perú, Brasil y Chile. Rio de Janeiro: E-papers, 2012.

TRAPNELL, Lucy. La experiencia del Programa de Formación de Maestros Bilingües de la Amazonía Peruana. En: MATO, Daniel (Coord.). Diversidad cultural e interculturalidad en educación superior. Experiencias en América Latina. Caracas: UNESCOIESALC, 2008. p. 403-412.

VALENZUELA, Estela Maris. Educación superior indígena en el Centro de Investigación y Formación para la Modalidad Aborigen (CIFMA): Génesis, desarrollo y continuidad. En: MATO, Daniel (Coord.). Instituciones interculturales de educación superior en América Latina. Procesos de construcción, logros, innovaciones y desafíos. Caracas: UNESCO-IESALC, 2009. p. 79-102.

YAPU, Mario. La experiencia de la Universidad Indígena Intercultural del Fondo Indígena, sede Universidad Mayor de San Simón, Bolivia En: MATO, Daniel (Coord.). Diversidad cultural e interculturalidad en educación superior. Experiencias en América Latina. Caracas: UNESCO-IESALC, 2008. p. 449-458. 